\title{
Traditional Chinese medicine combination therapy for patients with steroid- dependent ulcerative colitis: study protocol for a randomized controlled trial
}

\author{
Kai Zheng ${ }^{1,2+}{ }^{\text {, Hong Shen }}{ }^{2^{*}}$, Jia Jia ${ }^{3}$, Yuelin Lu², Lei Zhu ${ }^{4}$, Lu Zhang ${ }^{4}$ and Zhaofeng Shen ${ }^{4}$
}

\begin{abstract}
Background: Approximately $20 \%$ of patients with ulcerative colitis become steroid dependent. Azathioprine is recommended in steroid-dependent ulcerative colitis, but its side effects limit its use. Chinese herbal medicine has been widely used to treat ulcerative colitis in China. However, its effectiveness in steroid-dependent patients has not been evaluated. This study aims to investigate the efficacy of traditional Chinese medicine combination therapy with 5-aminosalicylic acid in patients with steroid-dependent ulcerative colitis.

Methods/Design: This is a parallel, multicenter, randomized controlled trial. One hundred and twenty eligible patients will be randomly assigned to a traditional Chinese medicine group or azathioprine group. All patients will be given basic treatment, which includes steroids and 5-aminosalicylic acid. Patients allocated to the traditional Chinese medicine group will receive basic treatment plus Chinese herbal medicine granules, while patients in the azathioprine group will receive basic treatment plus azathioprine. The whole study will last 24 weeks. The primary outcome measure is the steroid-free remission rate. Secondary outcome measures are health-related quality of life, efficacy of endoscopic response, degree of mucosal healing, and inflammation indicators.
\end{abstract}

Discussion: Results from this study may provide evidence for the effectiveness of traditional Chinese medicine combined with 5-aminosalicylic acid in patients with steroid-dependent ulcerative colitis. The findings will provide a basis for further confirmatory studies.

Trial registration: Chinese Clinical Trial Register, ChiCTR-IPR-15005760. Registered on 2 January 2015.

Keywords: 5-aminosalicylic acid, Azathioprine, Chinese herbal medicine, Steroid-dependent ulcerative colitis, Study protocol

\section{Background}

Ulcerative colitis is a form of inflammatory bowel disease characterized by a chronic relapsing clinical course. Intermittent rectal bleeding, diarrhea, and abdominal pain are often symptoms of ulcerative colitis. 5-Aminosalicylic acid is usually used in mild cases of ulcerative colitis. Steroids are another effective therapy for inducing remission in patients with moderate and severely active ulcerative colitis.

\footnotetext{
* Correspondence: jiangsutcm@163.com

Kai Zheng and Hong Shen contributed equally to this work as co-first author. ${ }^{\dagger}$ Equal contributors

${ }^{2}$ Department of Gastroenterology, Affiliated Hospital of Nanjing University of Chinese Medicine, Nanjing, Jiangsu 210029, China

Full list of author information is available at the end of the article
}

Approximately $20-34 \%$ of patients with ulcerative colitis have chronic active disease requiring several courses of steroids to achieve remission [1, 2]. Many patients initially benefit from steroid treatment but relapse shortly after treatment cessation or after dose reduction. Up to $20 \%$ of patients with ulcerative colitis become steroid dependent [3]. The European Crohn's and Colitis Organisation guidelines define "steroid-dependent" patients as those who are unable to reduce steroids below the equivalent of prednisolone $10 \mathrm{mg} /$ day within 3 months of starting steroid use, without recurrent active disease, or who have a relapse within 3 months of stopping steroid use [4]. The risks of long-term steroid therapy include osteoporosis, 
pathological fractures, cataracts, metabolic changes, psychological disturbances, and infection. Moreover, steroids have not been shown to be effective for the maintenance of remission.

The American College of Gastroenterology and European Crohn's and Colitis Organisation guidelines recommend that patients with steroid-dependent disease should be treated with azathioprine or 6-mercaptopurine $[5,6]$. Azathioprine is significantly more effective than 5 -aminosalicylic acid at achieving clinical and endoscopic remission in the treatment of steroid-dependent ulcerative colitis. In a single-center retrospective cohort study, 64 Asian patients with steroid-dependent inflammatory bowel disease were assessed, and $61 \%$ of patients were able to maintain clinical remission after 5 years of azathioprine therapy [7]. Toxicity induced by azathioprine and 6-mercaptopurine in patients with inflammatory bowel disease includes bone marrow suppression, drug-induced hepatitis, pancreatitis, and infection.

Complementary and alternative medicine use is common in patients with inflammatory bowel disease. The single most commonly used modality in most surveys is traditional Chinese medicine (TCM) [8]. Chinese herbal medicine is useful in improving symptoms, such as abdominal pain and diarrhea, and reducing inflammation in patients with ulcerative colitis. Many steroiddependent patients are dissatisfied with conventional therapies, including azathioprine's side effects; thus, they turn to TCM. However, it is not clear whether Chinese herbal medicine is effective for patients with steroiddependent ulcerative colitis. Therefore, the primary aim of our study is to evaluate the clinical efficacy of TCM treatment among patients with steroid-dependent ulcerative colitis. Health-related quality of life in steroiddependent patients will be a secondary aim.

\section{Methods/Design}

This is a multicenter, randomized controlled trial with two parallel groups and a 24-week follow-up. The centers include the Affiliated Hospital of Nanjing University of Chinese Medicine, Beijing Hospital of Chinese Medicine, Guangdong Province Hospital of Chinese Medicine, Longhua Hospital affiliated to Shanghai University of Chinese Medicine, the First Affiliated Hospital of Henan University of Chinese Medicine, Nanjing Drum Tower Hospital of Nanjing University of Medical School, Shengjing Hospital of China Medical University, the Hospital of Shanxi University of Chinese Medicine, the First Affiliated Hospital of Heilongjiang University of Chinese Medicine, and Nantong Hospital of Chinese Medicine. The study began in January 2015 and will last until October 2017. Trained researchers enroll participants. One hundred and twenty patients who meet the eligibility criteria will be recruited and allocated into two groups, with 60 individuals per group. This study has been approved by the Institutional Review Board of $\mathrm{Hu}$ man Research of the Affiliated Hospital of Nanjing University of Chinese Medicine (approval number: 2014NL073-03). Participants must provide written informed consent to participate in the study. All participants will continue their initial steroid and 5-aminosalicylic therapy, and will be randomly assigned to receive either Chinese herbal medicine or azathioprine. The overall flow of the trial is shown in Fig. 1.

\section{Eligibility criteria}

Participants will be considered for enrollment if they: range in age from 18 to 65 years; have a confirmed diagnosis of steroid-dependent ulcerative colitis; agree to participate in this clinical trial; and sign the informed consent form.

Patients will be excluded from the study if they: experience complications such as intestinal obstruction, intestinal perforation, toxic megacolon, or colorectal cancer; are pregnant, breast-feeding, or preparing for pregnancy; have an alanine aminotransferase level above the twice upper limit of normal; have a serum creatinine level above the upper limit of normal; have a platelet value of less than $50 \times 10^{9} / 1$; have a leukocyte count of less than $3.0 \times 10^{9} /$ l ; have a known history of alcohol or drug abuse; or are enrolled in other clinical trials.

\section{Sample size}

The remission rate for ulcerative colitis by azathioprine ranges from $59.1 \%$ to $87 \%[9,10]$, and the average rate is about $73 \%$. This is a noninferiority trial. For a two-sided significance level of 0.05 and power of $80 \%(\alpha=0.05, \beta$ $=0.2, \delta=0.15$ ), the sample size is calculated using the formula:

$$
n=12.365 \times P(1-P) / \delta^{2}
$$

Considering a $10 \%$ loss to follow-up, the sample size is 120 cases ( $n=60$ in each group).

\section{Randomization}

A randomization sequence was generated using SAS 9.4 (SAS Institute Inc., Cary, NC, USA). An independent person (Dr. Jiandong Zou) who is not involved in observation or assessment of the patients possesses the computer-generated randomization sequence. The researchers are blinded to the randomization sequence. The randomization procedure will be conducted by research assistants using an online computerized randomization system (https://sci.njpdkj.com/pdsci/). Eligible participants will be randomly assigned to either the TCM group or azathioprine group. 


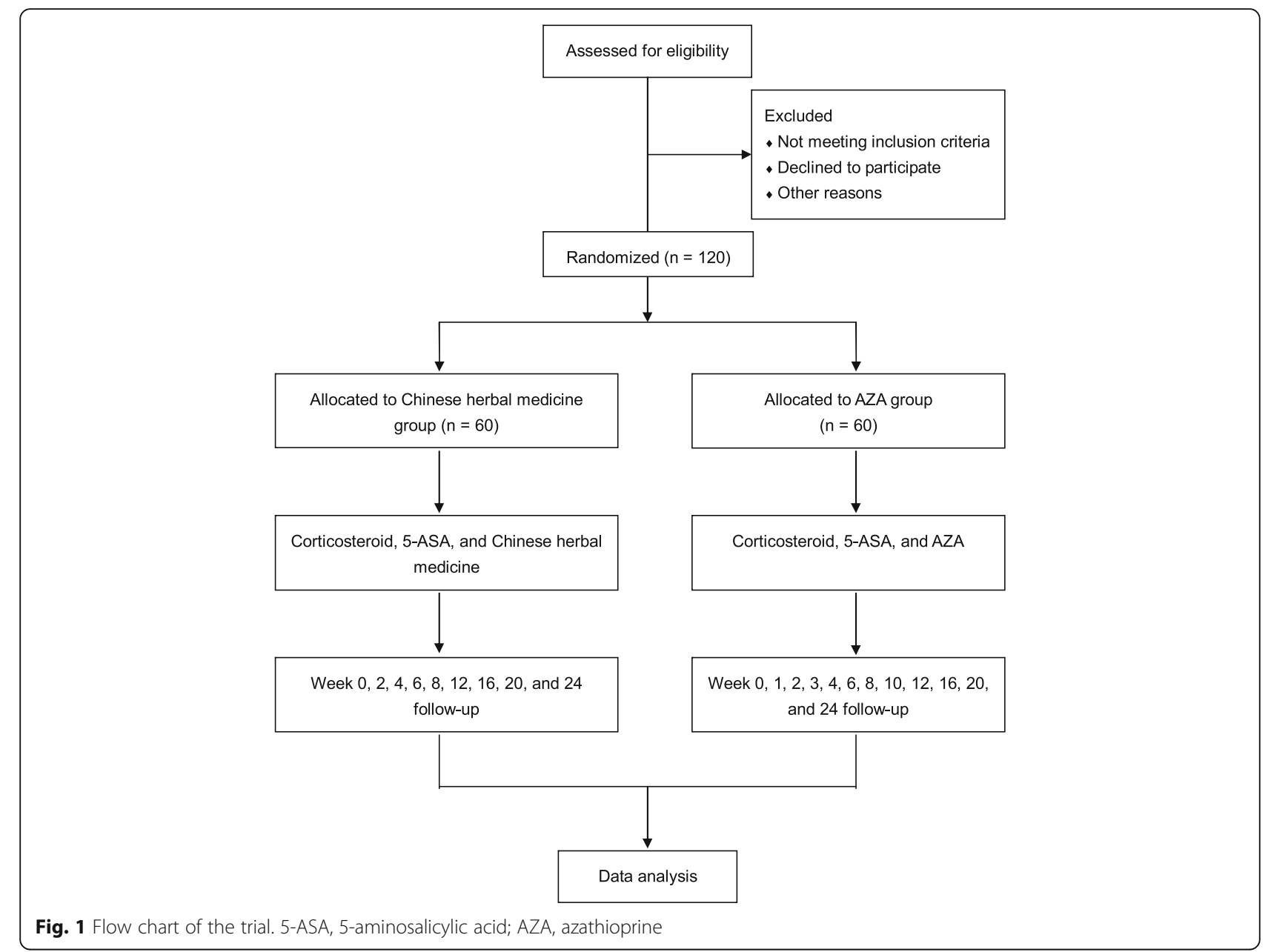

\section{Interventions}

\section{Basic treatment}

All eligible steroid-dependent patients will continue their initial steroid and 5-aminosalicylic therapy. Withdrawal of steroids occurs along with clinical remission. If there is clinical remission, a taper dose will be initiated with $5 \mathrm{mg}$ taper weekly until a $20 \mathrm{mg}$ daily dose is reached. Then tapering should proceed by $2.5 \mathrm{mg}$ per week [11].

\section{Chinese herbal medicine granules}

Patients in the TCM group will be treated with basic treatment plus granules. The granules include Radix Astragali (Huangqi) 20 g, Rhizoma Atractylodis Macrocephalae (Baizhu) $10 \mathrm{~g}$, Semen Coicis (Yiyiren) $20 \mathrm{~g}$, Rhizoma Dioscoreae (Shanyao) 20 g, Rhizoma Coptidis (Huanglian) 6 g, Radix Scutellariae (Huangqin) $10 \mathrm{~g}$, Radix Pulsatillae (Baitouweng) 10 g, Rhizoma Smilacis Glabrae (Tufuling) 15 g, Radix Angelicae Dahuricae (Baizhi) 12 g, Radix Paeoniae Alba (Baishao) 20 g, Pericarpium Citri Reticulatae (Chenpi) 12 g, Radix Saposhnikoviae (Fangfeng) 12 g, Radix Aucklandiae (Muxiang)
6 g, Radix Sanguisorbae (Diyu) 10 g, Radix Arnebiae (Zicao) 10 g, Radix et Rhizoma Rubiae (Qiancao) 20 g, Fructus Alpiniae Oxyphyllae (Yizhi) 10 g, Rhizoma Zingiberis Preparata (Paojiang) $6 \mathrm{~g}$, and Radix et Rhizoma Glycyrrhizae (Gancao) 6 g. All ingredients are manufactured as a Chinese herbal granule with a weight $27.5 \mathrm{~g}$ (Jiangyin Tianjiang Pharmaceutical Co., Ltd, Jiangyin, China). Patients will take one Chinese herbal medicine granule per day. The course of treatment is 24 weeks unless there is a loss of follow-up.

\section{Azathioprine}

The control group will take basic treatment plus azathioprine. Azathioprine treatment can be initiated at a dosage of $1 \mathrm{mg} / \mathrm{kg}$ per day $[12,13]$. The course of treatment will be in accordance with the TCM group.

\section{Outcome measures}

\section{Primary outcome measure}

The primary outcome measure of this study is the steroid-free remission rate in patients with steroiddependent ulcerative colitis. Remission is defined as a 
Mayo disease activity index score of $0-2$, with no individual subscore exceeding 1 [14]. The withdrawal time of steroids will also be recorded. The outcome measure will be evaluated at the end of 24 weeks. If there is no statistical significance between the steroid-free remission rate of the TCM group and the azathioprine group, then the mean withdrawal time from steroids will be compared.

\section{Secondary outcome measures}

The key secondary aim is to investigate the effect of Chinese herbal medicine granules on health-related quality of life in steroid-dependent patients compared with azathioprine, as measured by the inflammatory bowel disease questionnaire. Other secondary outcome measures include the efficacy of endoscopic response, which is defined as a decrease from baseline in a Mayo disease activity index endoscopy subscore by at least 1 [14]. The degree of mucosal healing is defined as a Mayo disease activity index endoscopy subscore of 0 or 1 [15]. Improvements in inflammation indicators include fecal calprotectin, $\mathrm{C}$ reactive protein, and erythrocyte sedimentation rate.

\section{Adverse events}

During the study, all adverse events, including toxicity and side effects, such as drug allergies, liver damage, renal failure, bone marrow suppression, and pancreatitis, will be reported and recorded in the case report form in detail. The researcher will report serious adverse events to the Institutional Review Board of Human Research of the Affiliated Hospital of Nanjing University of Chinese Medicine.

\section{Data management and statistical analysis}

Data recorded in printed case report forms are kept in locked cabinets. Data will be double-entered on electronic case report forms by two persons. Frequency, median, and mean \pm standard deviation of the steroid-free remission rate and the mean withdrawal time of steroids will be used for descriptive statistics. Student's $t$ test and analysis of variance will be employed using continuous variables with a normal distribution. The Mann-Whitney $U$ test and Kruskal-Wallis test will be used for continuous variables with abnormal distributions, such as health-related quality of life and inflammation markers. A chi-square test or Fisher's exact test will be used for categorical variables, including the efficacy of endoscopic response and the degree of mucosal healing. Results will be analyzed as per intention-to-treat. All data will be analyzed by an independent statistician using SAS 9.4. For all analyses, $P<0.05$ is considered statistically significant.

To summarize the present study protocol, please see Additional file 1 for an overview of enrollment, interventions and assessments, and Additional file 2 for an overview of the Standard Protocol Items: Recommendations for Interventional Trials (SPIRIT) checklist.

\section{Discussion}

Ulcerative colitis is a chronic inflammatory bowel disease characterized by a relapsing-remitting course owing to recurrent intestinal inflammation. Steroids are often prescribed for moderate to severe ulcerative colitis patients. Although steroids are usually effective, long-term steroid use should be avoided because of its severe side effects. Patients who initially respond to steroids but then relapse after tapering or shortly after discontinuation, and require reintroduction of steroids to induce remission are defined as steroid dependent. The therapeutic objective of steroid-dependent patients is to achieve steroid-free remission.

In view of the side effects of steroids, different drugs are used in the management of steroid-dependent ulcerative colitis. Drugs used include established agents, such as thiopurines, methotrexate, infliximab, adalimumab, vedolizumab, and golimumab. If common treatments fail, colectomy may be performed [16]. Current guidelines recommend thiopurines as first-line therapy to spare steroids in steroid-dependent ulcerative colitis $[3,6]$. Therefore, the thiopurine agents azathioprine and 6-mercaptopurine are used to sustain steroid withdrawal in patients with steroid-dependent ulcerative colitis [5]. Azathioprine is a purine analog that competitively inhibits the biosynthesis of purine ribonucleotides [17]. Azathioprine is the main therapy for steroiddependent patients in China, because vedolizumab and golimumab are not available, and adalimumab is not indicated for use in patients with ulcerative colitis. Furthermore, cessation of infliximab is still considered in clinical practice because of its high cost and fear of long-term side effects [18]. There is also a reduced response to infliximab, which may reach approximately $13 \%$ per year with uninterrupted scheduled maintenance therapy [19]. Fraser et al. reported that the remission rate was $87 \%$ in 346 ulcerative colitis patients who received more than 6 months of azathioprine treatment [10]. Another study demonstrated that the steroid-free remission rate was $59.1 \%$ in patients with steroiddependent or steroid-resistant ulcerative colitis treated with azathioprine [9]. The most common adverse event of azathioprine is leukopenia, which occurs in $3.8 \%$ of patients [20]. Other adverse events include hepatitis, infection, pancreatitis, and hair loss. Once absorbed into the plasma, azathioprine is nonenzymatically converted to 6-mercaptopurine. 6-Mercaptopurine can be metabolized to 6-methylmercaptopurine by the enzyme thiopurine methyltransferase (TPMT), to 6thiouric acid by the enzyme xanthine oxidase, and to 
the active metabolites of 6-thioguanine nucleotide. 6Thioguanine nucleotide is the active ribonucleotide of 6-mercaptopurine; it functions as a purine antagonist, resulting in immunosuppression and lymphocytotoxicity. A deficiency in TPMT may lead to preferential 6mercaptopurine metabolism into 6-thioguanine nucleotide, resulting in myelosuppression [21]. An apparent genetic polymorphism of TPMT is associated with TPMT enzyme deficiency. Despite a lower frequency of variant TPMT observed in Asian populations [22], lower starting doses of azathioprine are recommended in Asian populations than in white populations, along with close monitoring of complete blood count and liver function [13].

Chinese herbal medicine is a type of complementary and alternative medicine, and is often used in patients with inflammatory bowel disease [8], especially in China. Dai et al. [23] analyzed 247 patients with ulcerative colitis. The Sutherland Disease Activity Index scores for patients treated with TCM and those treated with integrated TCM and Western medicine were significantly lower for both groups after treatment $(P<0.01)$. Sugimoto et al. used the Chinese herbal medicine QingDai to treat 20 patients with moderate ulcerative colitis. The rates of clinical response, clinical remission, and mucosal healing were $72 \%, 33 \%$, and $61 \%$ at week 8 , respectively [24]. Qing-Dai stimulates mucosal type 3 innate lymphoid cells to generate interleukin-22, which induces antimicrobial peptide and tight junction production, suggesting a therapeutic mechanism for mucosal healing [25]. Our study using Chinese herbal medicine plus 5-aminosalicylic in patients with steroid-dependent ulcerative colitis aims to observe improvements in the primary outcome of steroid-free remission rate, and the secondary outcomes of health-related quality of life, endoscopic response, mucosal healing, and inflammation indicators. We expect that the steroid-free remission rate of the TCM group will be improved over that of the azathioprine group. The mean withdrawal time of steroids in the TCM groups should be less than that of the azathioprine group, which would reduce the risks of steroid therapy. We anticipate that the results will show that Chinese herbal medicine is effective for patients with steroid-dependent ulcerative colitis.

There are several limitations to our study. First, this is an open-label randomized controlled trial without double blinding. Because of the lack of placebo control groups, our findings will be interpreted with caution. Another limitation to this study is the lack of TPMT and 6-thioguanine nucleotide testing. Some patients do not respond to azathioprine because of lower 6-thioguanine nucleotide levels. Although a lower frequency of variant TPMT is observed in Asian populations, TPMT and 6thioguanine nucleotide testing may assist dose optimization of azathioprine to achieve response and avoid drug-induced toxicity. Both TPMT and 6thioguanine nucleotide testing will be determined in corollary studies. The last limitation is of first-choice therapy in steroid-dependent ulcerative colitis. The Toronto consensus in 2015 recommends anti-TNF therapy to induce and maintain complete steroid-free remission in patients with steroid-dependent ulcerative colitis [26]. The consensus group also recommends that anti-TNF plus azathioprine therapy is the preferred choice for steroid-dependent patients rather than monotherapy to induce complete remission [26, 27]. However, azathioprine is still the most practicable therapy for steroiddependent patients in China, because anti-TNF agents are not widely used. Combination therapy with anti-TNF agents will be available in the future. Notwithstanding its limitations, this study will suggest whether Chinese herbal medicine can be an effective complementary and alternative medicine in patients with steroid-dependent ulcerative colitis.

\section{Trial status}

The first patient in the study was enrolled on 24 April 2015. The trial is enrolling participants.

\section{Additional files}

Additional file 1: Schedule of enrollment, interventions, and assessments. (DOC $148 \mathrm{~kb}$ )

Additional file 2: SPIRIT 2013 Checklist: recommended items to address in a clinical trial protocol and related documents. (DOC $133 \mathrm{~kb}$ )

\section{Abbreviations \\ TCM: traditional Chinese medicine; TNF: tumor necrosis factor; TPMT: thiopurine methyltransferase}

Acknowledgements

We would like to thank Dr. Jiandong Zou for his support in developing the original study research proposal.

Funding

This study is supported by the Special Scientific Research for TCM of State Administration of Traditional Chinese Medicine of China (no. 201407001), and the National Natural Science Foundation of China (no. 81403343).

\section{Availability of data and materials}

Data sharing is not applicable to this article as no datasets were generated or analyzed during the current study.

\section{Authors' contributions}

$K Z$ and HS contributed to the design of the study protocol. $K Z, H S, Y L, L Z$, $L Z$, and $Z S$ participated in the project development. $K Z$ and $J J$ prepared the initial draft of the manuscript. KZ was involved in designing the statistical methods of the study design. KZ and JJ will be involved in the statistical analysis. HS is the project leader. All authors were responsible for the manuscript drafting and approved the final version.

\section{Competing interests}

The authors declare that they have no competing interests.

Consent for publication

Not applicable. 


\section{Ethics approval and consent to participate}

Ethical approvals for this 10-center study were obtained from the Institutional Review Board of Human Research of the Affiliated Hospital of Nanjing University of Chinese Medicine (Approval number: 2014NL-073-03), the ethics committee of Beijing Hospital of Chinese Medicine, the ethics committee of Guangdong Province Hospital of Chinese Medicine, the ethics committee of Longhua Hospital affiliated to Shanghai University of Chinese Medicine, the Institutional Review Board of Human Research of the First Affiliated Hospital of Henan University of Chinese Medicine, the ethics committee of Nanjing Drum Tower Hospital of Nanjing University of Medical School, the ethics committee of Shengjing Hospital of China Medical University, the ethics committee of the Hospital of Shanxi University of Chinese Medicine, the ethics committee of the First Affiliated Hospital of Heilongjiang University of Chinese Medicine, and the Institutional Review Board of Human Research of Nantong Hospital of Chinese Medicine. Informed consent will be obtained from all study participants. This study is registered with the Chinese Clinical Trial Registry, number ChiCTR-IPR-15005760.

\section{Author details}

${ }^{1}$ First Clinical Medical College, Nanjing University of Chinese Medicine, Nanjing, Jiangsu, China. 'Department of Gastroenterology, Affiliated Hospital of Nanjing University of Chinese Medicine, Nanjing, Jiangsu 210029, China. ${ }^{3}$ Department of Endocrinology, Affiliated Hospital of Nanjing University of Chinese Medicine, Nanjing, Jiangsu, China. ${ }^{4}$ Division of Scientific Research, Affiliated Hospital of Nanjing University of Chinese Medicine, Nanjing, Jiangsu, China.

Received: 18 August 2016 Accepted: 18 December 2016 Published online: 10 January 2017

\section{References}

1. Bianchi Porro G, Cassinotti A, Ferrara E, Maconi G, Ardizzone S. Review article: the management of steroid dependency in ulcerative colitis. Aliment Pharmacol Ther. 2007;26(6):779-94.

2. Faubion Jr WA, Loftus Jr EV, Harmsen WS, Zinsmeister AR, Sandborn WJ. The natural history of corticosteroid therapy for inflammatory bowel disease: a population-based study. Gastroenterology. 2001;121(2):255-60.

3. Armuzzi A, Pugliese D, Danese $\mathrm{S}$, et al. Infliximab in steroid-dependent ulcerative colitis: effectiveness and predictors of clinical and endoscopic remission. Inflamm Bowel Dis. 2013;19(5):1065-72.

4. Van Assche G, Dignass A, Panes J, et al. The second European evidencebased consensus on the diagnosis and management of Crohn's disease: definitions and diagnosis. J Crohns Colitis. 2010;4(1):7-27.

5. Kornbluth A, Sachar DB, Practice Parameters Committee of the American College of Gastroenterology. Ulcerative colitis practice guidelines in adults: American College Of Gastroenterology, Practice Parameters Committee. Am J Gastroenterol. 2010;105(3):501-23. Quiz, 524.

6. Dignass A, Van Assche G, Lindsay JO, et al. The second European evidencebased consensus on the diagnosis and management of Crohn's disease: current management. J Crohns Colitis. 2010;4(1):28-62.

7. Thia KT, Li M, Ling KL, Kong SC, Ooi CJ. Azathioprine is effective in corticosteroid-dependent Asian inflammatory bowel disease patients. Inflamm Bowel Dis. 2011;17(3):809-15.

8. Quattropani C, Ausfeld B, Straumann A, Heer P, Seibold F. Complementary alternative medicine in patients with inflammatory bowel disease: use and attitudes. Scand J Gastroenterol. 2003;38(3):277-82.

9. Kull E, Beau P. Compared azathioprine efficacy in ulcerative colitis and in Crohn's disease. Gastroenterol Clin Biol. 2002;26(4):367-71.

10. Fraser AG, Orchard TR, Jewell DP. The efficacy of azathioprine for the treatment of inflammatory bowel disease: a 30 year review. Gut. 2002;50(4):485-9.

11. Lichtenstein GR, Abreu MT, Cohen R, Tremaine W, Gastroenterological Association. American Gastroenterological Association Institute technical review on corticosteroids, immunomodulators, and infliximab in inflammatory bowel disease. Gastroenterology. 2006;130(3):940-87.

12. Ooi CJ, Fock KM, Makharia GK, et al. The Asia-Pacific consensus on ulcerative colitis. J Gastroenterol Hepatol. 2010;25(3):453-68.

13. Hibi T, Naganuma M, Kitahora T, Kinjyo F, Shimoyama T. Low-dose azathioprine is effective and safe for maintenance of remission in patients with ulcerative colitis. J Gastroenterol. 2003;38(8):740-6.
14. Sandborn WJ, Ghosh S, Panes J, et al. Tofacitinib, an oral Janus kinase inhibitor, in active ulcerative colitis. N Engl J Med. 2012;367(7):616-24.

15. Laharie D, Bourreille A, Branche J, et al. Ciclosporin versus infliximab in patients with severe ulcerative colitis refractory to intravenous steroids: a parallel, open-label randomised controlled trial. Lancet. 2012;380(9857):1909-15.

16. Khan HM, Mehmood F, Khan N. Optimal management of steroid-dependent ulcerative colitis. Clin Exp Gastroenterol. 2015;8:293-302.

17. Hibi T, Yajima T. A novel therapy for corticosteroid-dependent or corticosteroid-resistant patients with ulcerative colitis. Intern Med. 1997:36(5):317-8.

18. Louis E, Mary JY, Vernier-Massouille G, et al. Maintenance of remission among patients with Crohn's disease on antimetabolite therapy after infliximab therapy is stopped. Gastroenterology. 2012;142(1):63-70. e5. Quiz e31.

19. Gisbert JP, Panes J. Loss of response and requirement of infliximab dose intensification in Crohn's disease: a review. Am J Gastroenterol. 2009;104(3):760-7.

20. Connell WR, Kamm MA, Dickson M, Balkwill AM, Ritchie JK, Lennard-Jones JE. Long-term neoplasia risk after azathioprine treatment in inflammatory bowel disease. Lancet. 1994;343(8908):1249-52.

21. Kim MJ, Choe $\mathrm{YH}$. Monitoring and safety of azathioprine therapy in inflammatory bowel disease. Pediatr Gastroenterol Hepatol Nutr. 2013;16(2):65-70.

22. Krynetski EY, Evans WE. Genetic polymorphism of thiopurine Smethyltransferase: molecular mechanisms and clinical importance. Pharmacology. 2000;61(3):136-46.

23. Dai $Y C$, Zhang $Y L$, Wang $L$, et al. Clinical presentation and treatment strategies for ulcerative colitis: a retrospective study of 247 inpatients. Chin J Integr Med. 2016;22(11):811-6.

24. Sugimoto S, Naganuma M, Kiyohara $H$, et al. Clinical efficacy and safety of oral Qing-Dai in patients with ulcerative colitis: a single-center open-label prospective study. Digestion. 2016;93(3):193-201.

25. Sugimoto S, Naganuma M, Kanai T. Indole compounds may be promising medicines for ulcerative colitis. J Gastroenterol. 2016;51(9):853-61.

26. Bressler B, Marshall JK, Bernstein CN, et al. Clinical practice guidelines for the medical management of nonhospitalized ulcerative colitis: the Toronto consensus. Gastroenterology. 2015;148(5):1035-58. e3.

27. Panaccione R, Ghosh S, Middleton S, et al. Combination therapy with infliximab and azathioprine is superior to monotherapy with either agent in ulcerative colitis. Gastroenterology. 2014;146(2):392-400. e3.

\section{Submit your next manuscript to BioMed Central and we will help you at every step:}

- We accept pre-submission inquiries

- Our selector tool helps you to find the most relevant journal

- We provide round the clock customer support

- Convenient online submission

- Thorough peer review

- Inclusion in PubMed and all major indexing services

- Maximum visibility for your research

Submit your manuscript at www.biomedcentral.com/submit
Biomed Central 